demographics; barriers of use for available STI POCTs; characteristics of an ideal POCT, including prioritising pathogens for targets; and building your own POCT". Practicing clinicians and academic experts from two venues, STI-related international conference attendees and U.S. STD clinic clinicians, were invited to participate in the clinician survey. Professionals from industry in the STI diagnostic field were invited to participate in the industry survey. $\chi^{2}$ test and conditional logistical regression were used for data analysis.

Results Clinician survey participants $(n=218)$ identified "the time frame required" (39.9\%), "complexity" (31.2\%), and "interruption of work flow" (30.3\%) as the top three barriers making it difficult to use STI POCTs, while the industry survey participants $(n=107)$ identified "complexity" (65.4\%), "unreliability" (53.3\%), and "difficulty in reading results" (34.6\%) as the top three barriers. Significant differences in barriers named in the two surveys included "complexity", "the time frame required", "laboratory driven", "difficulty in reading results", and "unreliability". Participants from both surveys ranked $C$ trachomatis as the top priority organism chosen for a new POCT (clinician: 62\%, industry: 39\%, p<0.05), followed by a test that would diagnose early seroconversion for HIV (clinician: $14 \%$, industry: $32 \%, \mathrm{p}<0.05$ ). Sensitivity was always the most important attribute to be considered for a new STI POCT by both participant groups. Participants of the clinician group chose cost as the second priority attribute, while those of the industry group chose specificity as the second priority.

Conclusions We identified differences in the perceptions regarding barriers and ideal attributes for STI POCTs between frontline clinical providers and industry personnel. Tailored training is warranted to inform scientists, biomedical engineers, and other industry experts about characteristics that clinicians desire for STI POCTs

\section{3-S6.06 SELF-COLLECTION VS PHYSICIAN-COLLECTION FOR THE DETECTION OF CHLAMYDIA TRACHOMATIS, NEISSERIA GONORRHOEAE, TRICHOMONAS VAGINALIS AND MYCOPLASMA GENITALIUM}

\section{doi:10.1136/sextrans-2011-050109.138}

\begin{abstract}
${ }^{1} \mathrm{~J}$ Kwatampora, ${ }^{2} \mathrm{~V}$ Senkomago, ${ }^{3} \mathrm{~N}$ Mugo, ${ }^{4} \mathrm{C}$ Hill, ${ }^{2} \mathrm{~J}$ Ting, ${ }^{5} \mathrm{M}$ Chitwa, ${ }^{1} \mathrm{~S}$ Patel, ${ }^{1} \mathrm{H}$ Gukare, ${ }^{1} \mathrm{~J}$ Kimani, ${ }^{2} \mathrm{~J}$ Smith. ${ }^{1}$ University of Nairobi/University of Manitoba STI Collaboration Nairobi, Kenya; ${ }^{2}$ University of North Carolina at Chapel Hill, Chapel Hill, USA; ${ }^{3}$ University of Nairobi/University of Manitoba STI Collaboration, Nairobi, Kenya; ${ }^{4}$ Gen-Probe Incorporated, San Diego, CA. San Diego, USA; ${ }^{5}$ University of Nairobil University of Manitoba STI Collaboration, Nairobi, Kenya
\end{abstract}

Background To determine the efficacy of self-collection for primary screening of an array of sexually transmitted infections.

Methods A cross-sectional baseline analysis was conducted on a cohort of 300 female sex workers (FSW) within an outpatient clinic from a suburban/slum area of Nairobi, from December 2009 to June 2010. APTIMA transcription-mediated amplification (TMA) assays (Gen-Probe Incorporated) were used for the detection of Chlamydia trachomatis (CT), Neisseria gonorrhoeae (GC), Trichomonas vaginalis (Trich) and Mycoplasma genitalium (MGen) infections and, were analysed in San Diego, California. The APTIMA COMBO 2 assay (AC2) was used for CT/GC, the APTIMA Trichomonas vaginalis assay for Trich, and an APTIMA Mycoplasma genitalium research assay for Mgen. FSW conducted a self-collected sample in privacy, based on standardised instructions, and then underwent a pelvic exam to obtain a physician-collected sample.

Results A total of 299 FSW (mean age of 30) participated, of which $15 \%$ were HIV-seropositive. MGen was the most common infection
(12.7\% in physician; $20.7 \%$ in self-collection), followed by Trich $(7.7 \% ; 9.7 \%)$, CT $(4.3 \% ; 5.4 \%)$, and GC $(2.7 \% ; 3.3 \%)$. For all STIs examined, the self-collected samples detected more positive cases than the physician-collected samples. No self-collected sample missed an infection that was picked by the physician sample. $\kappa$ for agreement between self-collected and physician-collected samples were high for CT: 0.89, GC: 0.89 and Trich: 0.87 , and slightly lower for MGen: 0.73 . Using physician sampling as the gold-standard, the sensitivity of self-collection was $100 \%$ for all STIs. The specificity for self-collection was high for CT, GC, and Trich (99.0\%, 99.3\% and 97.8\% respectively) and slightly lower for MGen (91.6\%).

Conclusion A single self-collected sample appeared to perform with comparable sensitivity and specificity to that of physician-collected sampling for the detection of Chlamydia trachomatis, Neisseria gonorrhoeae, Trichomonas vaginalis and Mycoplasma genitalium.

\section{Basic sciences oral session 1-Genomics, replication and pathogenesis \\ 04-S1.01 RAPID ARRAY-BASED MULTILOCUS GENOTYPING OF CHLAMYDIA TRACHOMATIS: THE EASY AND ECONOMICAL ALTERNATIVE}

doi:10.1136/sextrans-2011-050109.139

${ }^{1} \mathrm{~B}$ Herrmann, ${ }^{1} \mathrm{~L}$ Christerson, ${ }^{2} \mathrm{~A}$ Ruettger, ${ }^{3} \mathrm{~K}$ Gravningen, ${ }^{4} \mathrm{R}$ Ehricht, ${ }^{2} \mathrm{~K}$ Sachse. ${ }^{1}$ Uppsala University Uppsala, Sweden; ${ }^{2}$ Friedrich-Loeffler-Institut Federal Research Institute for Animal Health Jena, Germany; ${ }^{3}$ University Hospital of Northern Norway, Tromsø, Norway; ${ }^{4}$ Alere Technologies GmbH Jena, Germany

Background Genotyping ofChlamydia trachomatis (C.) is an important technique to understand its epidemiology. Methods such as DNA sequencing of the ompA gene or multilocus sequence typing (MLST), either offer limited epidemiological resolution, or are laborious and expensive, or both. Here we present a microarraybased method for genotyping of $C$ trachomatis.

Methods The database for our high-resolution MLST system (http:// mlstdb.bmc.uu.se/) was used to design a multilocus typing (MLT) DNA microarray based on the ArrayStrip format (Alere Technologies, Jena, Germany). In total, the present MLT array version includes 210 different oligonucleotide probes covering the discriminatory variation in the highly variable, but stable, MLST target regions (hctB, CT058, CT144, CT172 and pbpB), as well as 61 probes for ompA. Validation of the array was done by examining 80 clinical C. trachomtis specimens from unselected adolescents and compare with results from MLST and ompA-based serotyping.

Results Successful typing was achieved for 78 (97\%) of the specimens. Processing of the obtained hybridisation patterns resulted in 17 different MLT array groups, whereas sequence-based examination led to 19 MLST genotypes and seven ompA genotypes. Thus, the MLT microarray assay provided 2.4 times higher resolution than ompA and separated the commonly predominating ompA E/Bour genotype into seven genotypes. The MLT array showed $100 \%$ specificity. Compared to MLST analysis, the equipment needed for the MLT array is about $75 \%$ cheaper, consumables are $50 \%$ cheaper, analysis can be completed within one working day, instead of 3-4 days, and data analysis is easily conducted in high troughput conditions using up to 96 wells, while the practical operations are easy-to-handle and do not require specially trained personnel.

Conclusion This novel MLT array is a promising alternative for high resolution and high throughput typing of C. trachomatis and will facilitate molecular epidemiology studies of chlamydia infections. 